2001;24:214-218). (Respond: Dr Libensen, Division of Pediatric Neurology, Floating Hospital for Children at New England Medical Center \#330, 750 Washington Street, Boston, MA 02111).

COMMENT. Valproate, and to a lesser degree carbamazepine, are superior to phenobarbital in completely suppressing interictal EEG seizure activity in children with epilepsy. Whereas VPA and CBZ are equally effective in controlling generalized discharges, VPA is superior to both CBZ and PHB in suppressing focal EEG discharges, especially when the interval between EEGs is less than 1 year. VPA is also superior to ethosuximide in suppressing generalized epileptiform discharges in children with absence seizures (personal observation).

\title{
GABAPENTIN ADD-ON THERAPY FOR PARTIAL SEIZURES
}

The efficacy and safety of gabapentin (GBP) as add-on therapy in 237 children, aged 3 to 12 years, with refractory partial seizures were studied at the Alder Hey Children's Hospital, Liverpool, UK. In a multicenter, open-label trial over a 6 month period, efficacy of GBP was evaluated by comparing the frequency of partial seizures during a 6-12 week base-line phase of a previous double-blind study to the frequency during the follow-up study. The median percent decrease in seizure frequency was $34 \%$ and the overall response rate $>50 \%$ reduction in seizures) was $34 \%$. Six percent of patients withdrew because of adverse events, and $20 \%$ because of lack of efficacy. Concurrent AEDs were maintained in 78\%; doses were decreased in $11 \%$ and increased in $11 \%$. (Appleton R, Fichtner K, LaMoreaux L et al. Gabapentin as add-on therapy in children with refractory partial seizures: a 24-week, multicentre, open-label study. Dev Med Child Neurol April 2001;43:269-273). (Respond: Dr Richard Appleton, Alder Hey Children's Hospital, Liverpool L12 2AP, UK).

COMMENT. Gabapentin is well tolerated and effective as add-on treatment for refractory partial seizures in children.

\section{LANGUAGE DISORDERS}

\section{LANDAU-KLEFFNER SYNDROME: COURSE AND OUTCOME}

The presentation, course and outcome of Landau-Kleffner syndrome (LKS) were studied in 18 children (11 girls, 7 boys) followed for a mean of 67 months at Guy's Hospital, London, UK. All showed receptive language regression and electrical status epilepticus in sleep (ESES). Mean age at onset was 4 years 9 months (range 25-84 months). The length of ESES (mean 44 months) was correlated significantly with length of period between onset of illness and onset of recovery, with length of daytime EEG abnormalities, seizure frequency, and period of seizures (mean 36 months). The correlation between language outcome and seizure activity was weaker than that with ESES. Receptive language outcomes for those whose ESES lasted $<3$ years was significantly better than those with ESES for $>3$ years. Behavior problems (ADHD and ODD) were common (50\%), especially in patients with frontal lobe EEG abnormalities. Behavior abnormality in the acute phase was not correlated with seizure frequency, length of ESES, length of daytime EEG abnormality, nor with subsequent language outcome; there was a mild association with lower IQ. All children had impaired short-term memory at follow-up. Recovery began at a mean age of 106 months, and the mean duration of acute involvement was 50 months. Receptive and expressive language outcomes were strongly correlated, but language and IQ measures showed weaker associations. Language outcome was normal in three. In children with ESES 